\title{
Effects of sports participation on psychiatric symptoms and brain activations during sports observation in schizophrenia
}

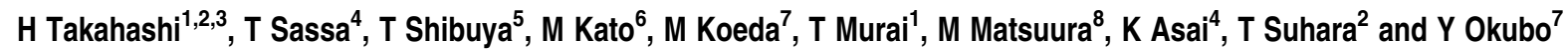

Weight gain has been identified as being responsible for increased morbidity and mortality rates of schizophrenia patients. For the management of weight gain, exercise is one of the most acknowledged interventions. At the same time, exercise and sports have been recognized for their positive impact on psychiatric symptoms of schizophrenia. However, the neurobiological basis for this remains poorly understood. We aimed to examine the effect of sports participation on weight gain, psychiatric symptoms and brain activation during sports observation in schizophrenia patients. Thirteen schizophrenia patients who participated in a 3-month program, including sports participation and 10 control schizophrenia patients were studied. In both groups, body mass index (BMI), Positive and Negative Syndrome Scale (PANSS), and brain activation during observation of sports-related actions measured by functional magnetic resonance imaging were accessed before and after a 3-month interval. BMI and general psychopathology scale of PANSS were significantly reduced in the program group but not in the control group after a 3-month interval. Compared with baseline, activation of the body-selective extrastriate body area (EBA) in the posterior temporal-occipital cortex during observation of sports-related actions was increased in the program group. In this group, increase in EBA activation was associated with improvement in the general psychopathology scale of PANSS. Sports participation had a positive effect not only on weight gain but also on psychiatric symptoms in schizophrenia. EBA might mediate these beneficial effects of sports participation. Our findings merit further investigation of neurobiological mechanisms underlying the therapeutic effect of sports for schizophrenia.

Translational Psychiatry (2012) 2, e96; doi:10.1038/tp.2012.22; published online 20 March 2012

\section{Introduction}

Obesity and weight gain were identified as factors responsible for increased morbidity and mortality rates of patients with schizophrenia long before the introduction of second-generation antipsychotics. ${ }^{1,2}$ With their introduction, awareness of weight gain has increased considerably among many healthcare professionals and patients. ${ }^{3-5}$ For the management of obesity and weight gain, exercise is one of the most acknowledged interventions. ${ }^{6,7}$ At the same time, obesity was associated with negative effects on memory in schizophrenia, ${ }^{8}$ and exercise/sports have been recognized to have a positive impact on cognition and psychiatric symptoms of schizophrenia. $^{9,10}$ Recent neuroscience research has presented increasing evidence concerning the neurobiological mechanism involved in the positive effect of exercise on cognition in the general population. ${ }^{11}$ In addition, a recent study has demonstrated that exercise induced an increase in hippocampal volume and improvement in short-term memory in schizophrenia. ${ }^{12}$ However, the neurobiological basis of the positive impact of exercise on psychiatric symptoms of schizophrenia remains poorly understood.

Although multiple mechanisms are likely involved in this positive impact of exercise, one possible mechanism is the interaction between action execution and action perception. It is widely documented that the systems that mediate action perception, planning and execution are overlapping and interact with each other, ${ }^{13-16}$ and that the mirror neuron system (MNS) has a crucial role in this interaction. ${ }^{17}$ MNS is suggested to contribute to anticipating and predicting others' behaviors during social interactions, ${ }^{18}$ and its dysfunction might be related to some aspects of psychiatric symptoms of schizophrenia. ${ }^{19}$

We demonstrated decreased activation of the bodyselective extrastriate body area (EBA) in the posterior temporal-occipital cortex during observation of sports-related actions in schizophrenia. ${ }^{20}$ Originally, EBA was identified as an area that responds selectively to static human bodies and body parts. ${ }^{21}$ However, recent studies have suggested an

\footnotetext{
${ }^{1}$ Department of Psychiatry, Kyoto University Graduate School of Medicine, Kyoto, Japan; ${ }^{2}$ Department of Molecular Neuroimaging, National Institute of Radiological Sciences, Chiba, Japan; ${ }^{3}$ Precursory Research for Embryonic Science and Technology (PRESTO), Japan Science and Technology Agency, Saitama, Japan; ${ }^{4}$ Department of Psychiatry, Asai Hospital, Togane, Japan; ${ }^{5}$ Department of Human Science, Toyo Gakuen University, Tokyo, Japan; ${ }^{6}$ Department of Neuropsychiatry, Keio University School of Medicine, Tokyo, Japan; ${ }^{7}$ Department of Neuropsychiatry, Nippon Medical School, Tokyo, Japan and ${ }^{8}$ Department of Life Sciences and Bio-informatics, Graduate School of Health Sciences, Tokyo Medical and Dental University, Tokyo, Japan

Correspondence: Professor H Takahashi, Department of Psychiatry, Kyoto University Graduate School of Medicine, 54 Shogoin-Kawara-cho, Sakyo-ku, Kyoto 606-8507, Japan.
}

E-mail: hidehiko@ kuhp.kyoto-u.ac.jp

Keywords: exercise; extrastriate body area; fMRI; schizophrenia; weight gain

Received 19 January 2012; revised 6 February 2012; accepted 14 February 2012 
extended role for EBA as a part of the system for understanding others' behaviors, involving not only static visual perception of body parts but also the planning, imagination and execution of actions. ${ }^{13,22,23}$ Previous neuroimaging studies in healthy subjects showed that motor experience/ expertise increased the activation of MNS during the observation of experienced movements. ${ }^{24,25}$ Thus, in addition to the expected effect of weight loss, we hypothesized that sports participation would have a positive effect on EBA activation during sports observation in chronic schizophrenia and psychiatric symptoms, and especially on the negative and general psychopathology scale of the Positive and Negative Syndrome Scale (PANSS).

\section{Materials and methods}

Participants. A three-month program primarily aiming to manage weight gain in chronic schizophrenia patients was implemented at the day-hospital unit of Asai Hospital. The multidisciplinary program team consisted of a group of professionals from diverse disciplines (physical therapists, psychiatric nurses, psychiatrists, nutritionist, and pharmacist). The program consisted of a series of modules, including exercise, nutrition education and medication counseling. The exercise module was further composed of aerobic exercise (walking and jogging), muscle-stretching exercise (static muscle-stretching for the whole body and dynamic muscle-stretching for the arms and legs; two repetitions), and sports exercise (basketball). The exercise module was 30-60 min long, and was delivered twice a day (a total of 60-120 min per day) from Monday to Saturday. Because the program was conducted in the summer season, its duration was determined by weather condition (temperature and humidity) and the participants' physical fatigue. For each participant, the exercise intensity level was set at 11-13 (fairly light-somewhat hard) of the Borg scale (rating of perceived exertion). ${ }^{26}$ Although the Borg scale is recommended by the American College of Sports Medicine as a tool to define the intensity of the exercise intervention, ${ }^{27}$ it should be noted that it is a subjective method. The program contents were announced through brochures, and 13 patients with schizophrenia, meeting the Diagnostic and Statistical
Manual of Mental Disorders, 4th Edition (DSM-IV criteria for schizophrenia, ${ }^{28}$ participated in the program. Another 10 schizophrenia patients who were attending the day-hospital unit of Asai Hospital but not the program were studied as controls. The patients' demographics (age, gender, body mass index (BMI), illness duration, and daily chlorpromazineequivalent antipsychotics dosage $\mathrm{e}^{29,30}$ ) of both the program and control groups are shown in Table 1.

Diagnoses were based on the patient edition of the Structured Clinical Interview for DSM-IV Axis I Disorders ${ }^{31}$ conducted by trained senior psychiatrists. Exclusion criteria were current or past substance abuse and a history of alcoholrelated problems, mood disorder, or organic brain disease. All of the patients were right-handed, and they all underwent magnetic resonance imaging to rule out cerebral anatomic abnormalities. All patients were using the day-hospital unit of Asai Hospital. After complete explanation of the study, written informed consent was obtained from all participants, and the study was approved by the Ethics Committee of Asai Hospital.

Immediately before and after the three-month program, body mass index (BMI), clinical symptoms and functional magnetic resonance imaging (fMRI) were assessed. For the control group, the same measures were collected before and after a 3-month interval. All patients received antipsychotics, and their medications remained unchanged during this study. Clinical symptoms were assessed by the PANSS for schizophrenia. $^{32}$ The ratings were reviewed by trained senior psychiatrists after patient interviews, and disagreements were resolved by consensus; consensus ratings were used in this study.

Materials. We used the same visual stimuli as in the previous reports. ${ }^{20,23}$ Two types of video clips were provided (basketball-related motions (BRM) and basketballunrelated motions (BUM)). Examples of the video clips are shown in Figure 1. BRM consisted of three types of scenes (player dribbling, two players performing man-to-man defense/offense and player shooting a free throw). BUM also consisted of three types of scenes (player carrying a basketball, one person crossing in front of another without interaction and player rolling a basketball). To make BRM and BUM as similar as possible, all players in the video clips

Table 1 Demographic values for subjects, measures of body mass index and PANSS at baseline and after 3-month program or 3-month interval

\begin{tabular}{|c|c|c|c|c|}
\hline & \multicolumn{4}{|c|}{ Mean (s.d.) } \\
\hline & \multicolumn{2}{|c|}{ Program group } & \multicolumn{2}{|c|}{ Control group } \\
\hline & Baseline & Outcome & Baseline & Outcome \\
\hline Age (years) & $43.5(11.8)$ & - & $39.9(13.6)$ & - \\
\hline Gender (male/female) & $7 / 6$ & - & $5 / 5$ & - \\
\hline Illness duration (years) & $22.1(15.0)$ & - & $14.8(11.7)$ & - \\
\hline Daily antipsychotics dosage (mg) & $506.8(426.6)$ & - & $592.0(390.3)$ & - \\
\hline Body mass index & $28.5(4.7)$ & $27.8(4.3)$ & $26.3(3.5)$ & $26.5(4.5)$ \\
\hline Positive scale of PANSS & $15.8(4.2)$ & $15.5(3.6)$ & $13.1(2.5)$ & $13.6(3.3)$ \\
\hline Negative scale of PANSS & $20.7(5.3)$ & $19.8(5.1)$ & $20.8(4.1)$ & $21.0(3.7)$ \\
\hline General psychopathology scale of PANSS & $37.4(6.7)$ & $35.0(7.3)$ & $35.4(3.1)$ & $35.8(3.5)$ \\
\hline Total score of PANSS & $73.9(15.0)$ & $70.3(14.8)$ & $69.3(4.7)$ & $70.4(5.5)$ \\
\hline
\end{tabular}

Abbreviation: PANSS, Positive and Negative Syndrome Scale. 


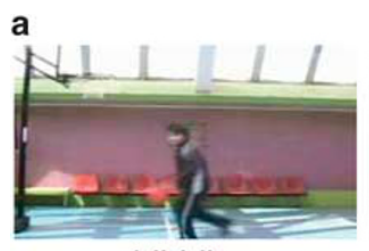

dribbling

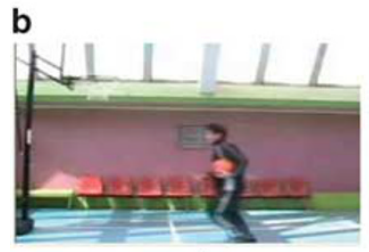

carrying

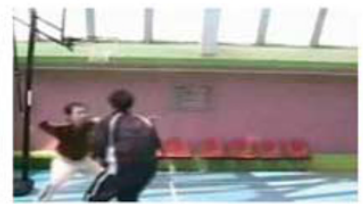

man-to-man

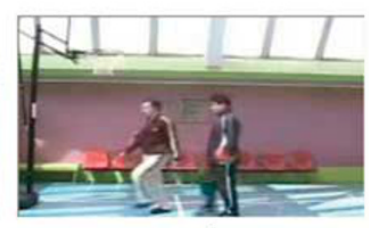

crossing

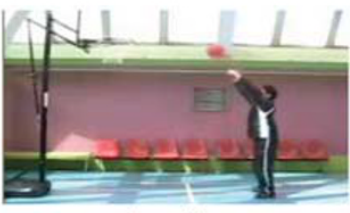

shooting

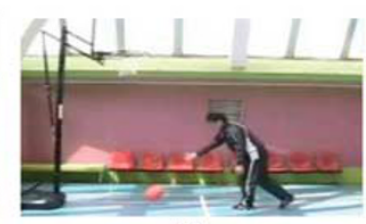

rolling

Figure 1 Sample of still frames from video clips. (a) Basketball-related motions and (b) basketball-unrelated motions.

performed in front of a basket goal on a basketball court, and the number of persons, objects, motion direction and speed were matched, that is, rolling a basketball, carrying a basketball, and crossing in front of another without interaction corresponded to shooting a free throw, dribbling, and man-to-man defense, respectively. The video clips were projected via computer and telephoto lens onto a screen mounted on a head-coil. The subjects were instructed to pay attention to the video clips and to press a selection button with the right index finger when they watched the free-throw scene and the basketball-rolling scene, indicating that they had paid attention to them. The experimental design consisted of five blocks for each of the two conditions (BRM and BUM) interleaved with 20 -s rest periods. The order of the two conditions was randomized. During the rest condition, participants viewed a crosshair pattern projected to the center of the screen. In the BRM and BUM 24-s blocks, three scenes were presented twice for $4 \mathrm{~s}$ each.

Image acquisition. Images were acquired with a 1.5. Tesla Signa system (General Electric, Milwaukee, WI, USA). Functional images of 115 volumes were acquired with $\mathrm{T}^{*}$ weighted gradient echo planar imaging sequences sensitive to blood oxygenation level-dependent contrast. Each volume consisted of 40 transaxial contiguous slices with a slice thickness of $3 \mathrm{~mm}$ to cover almost the whole brain (flip angle, $90^{\circ}$; echo time (TE), $50 \mathrm{~ms}$; repetition time (TR), $4 \mathrm{~s}$; matrix, $64 \times 64$; field of view, $24 \times 24 \mathrm{~cm}$ ). High-resolution, T1-weighted anatomic images were acquired for structure images (124 contiguous axial slices, 3D Spoiled-Grass sequence, slice thickness $1.5 \mathrm{~mm}$, TE, $9 \mathrm{~ms}$; TR, $22 \mathrm{~ms}$; flip angle, $30^{\circ}$; matrix, $256 \times 192$; field of view, $25 \times 25 \mathrm{~cm}$ ).

Analysis of functional imaging data. Data analysis was performed with statistical parametric mapping software package (SPM05) (Wellcome Department of Cognitive Neurology, London, UK) running with MATLAB (Mathworks, Natick, MA, USA). All volumes were realigned to the first volume of each session to correct for subject motion and were spatially normalized to the standard space defined by the Montreal Neurological Institute template. After normalization, all scans had a resolution of $2 \times 2 \times 2 \mathrm{~mm}^{3}$. Functional images were spatially smoothed with a 3D isotropic Gaussian kernel (full width at half maximum of $8 \mathrm{~mm}$ ). Low frequency noise was removed by applying a high-pass filter (cutoff period =192 s) to the fMRI time series at each voxel. A temporal smoothing function was applied to the $\mathrm{fMRI}$ time series to enhance the temporal signal-to-noise ratio. Significant hemodynamic changes for each condition were examined using the general linear model with boxcar functions convolved with a hemodynamic response function. Statistical parametric maps for each contrast of the t-statistic were calculated on a voxel-by-voxel basis.

To assess the specific condition effect, we used the contrasts of BRM minus BUM. A random effects model, which estimates the error variance for each condition across the subjects, was implemented for group analysis. This procedure provides a better generalization for the population from which data are obtained. The contrast images were obtained from single-subject analysis and entered into the group analysis. We compared BRM-minus-BUM contrasts before and after the program (or 3-month interval for control group) with paired t-tests. Significant differences were recognized at a height threshold of $P<0.001$, uncorrected, and an extent threshold of five contiguous voxels.

\section{Results}

Clinical data (BMI and PANSS). All participants completed the program. Clinical data from before and after a 3-month interval are shown in Table 1. There were no significant differences between the two groups in terms of age $(P=0.51)$, illness duration $(P=0.21)$, and daily antipsychotics dosage $(P=0.62)$. Two-way repeated-measures ANOVA with subject groups (program group, control group) as a between-subjects factor and time (before and after a 3-month interval) as within-subjects factors revealed that there were significant group $\times$ time interactions in $\mathrm{BMI}$ $\left(F_{1,21}=5.0, P=0.04\right)$ and general psychopathology scale of 

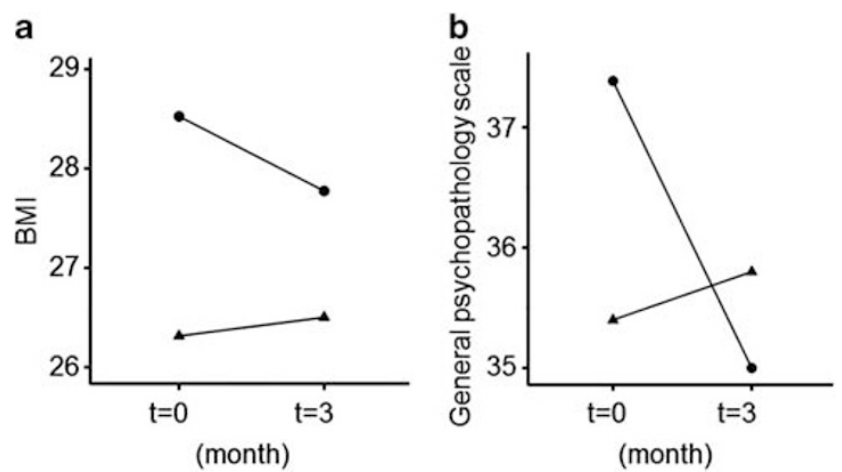

Figure 2 Changes between baseline and end point in (a) BMl and (b) general psychopathology scale in the two groups. - and $\boldsymbol{\Delta}$ indicate the mean of the program group $(n=13)$ and control $(n=10)$ group, respectively.

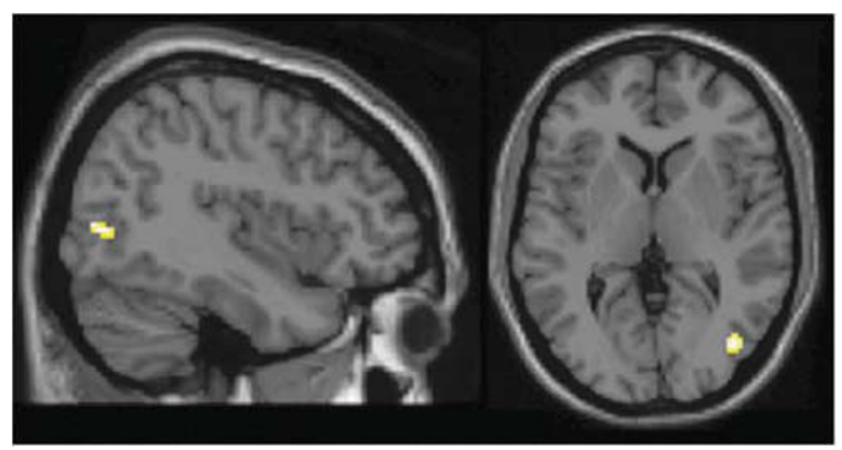

Figure 3 Images showing brain activation difference before and after the program. After the program, greater EBA activation was observed in BRM minus BUM condition compared with baseline in program group $(n=13)$.

PANSS $\left(F_{1,21}=19.4, \quad P<0.001\right)$. Post-hoc paired $t$-tests revealed that $\mathrm{BMI}$ and general psychopathology scale were significantly reduced in the program group $(P<0.001$, $t=3.84$, df $=12$ and $P<0.001, t=4.65$, df $=12)$ but not in the control group $(P=0.68, t=-0.46$, df $=9$ and $P=0.17$, $t=-1.50, \mathrm{df}=9$, respectively) (Figure 2). There was no significant group $\times$ time interaction in positive and negative scale. No main effect of group was found in BMI, positive, negative and general psychopathology scale $(P>0.05)$. No main effect of time was found in BMI, positive and negative scale $(P>0.05)$, except for general psychopathology scale $\left(\mathrm{F}_{1,21}=9.85, P=0.005\right)$.

fMRI result. All patients paid attention to the video clips and pressed the button appropriately (accuracy was virtually $100 \%$ ).

In the program group, BRM-minus-BUM condition after the program showed significantly greater right EBA activation (peak: $x=42, y=-74, z=4, Z$ score $=4.12$, cluster size: 43 voxels) compared with that before the program (Figure 3 ). In the control group, there was no significant difference in brain activation of BRM-minus-BUM condition before and after a 3-month interval. BRM-minus-BUM condition after the program did not show reduced regional brain activation compared with that before the program in both groups.

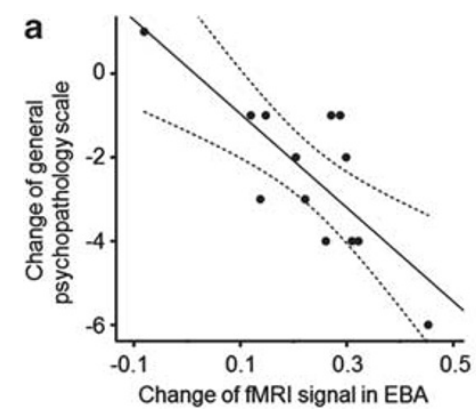

b

Figure 4 Correlation between the fMRI signal changes in EBA and changes in the general psychopathology scale of PANSS. (a) Plots and regression line of correlation between the fMRI signal changes and changes in the general psychopathology scale. Dashed lines are 95\% confidence interval boundaries. (b) Image showing the result of confirmatory SPM regression analysis.

We conducted regression analyses to demonstrate a link between brain activation change before and after the program and clinical data (PANSS scale and BMI) changes before and after the program in the program group. Using the eigenvariate of the EBA cluster revealed by comparison between before and after the program, we plotted the $\mathrm{FMRI}$ signal changes and changes in PANSS scores.

In the program group, the $\mathrm{fMRI}$ signal changes in EBA were negatively correlated with changes in the general psychopathology scale of PANSS $(r=-0.78, P=0.002)$ (Figure 4). That is, patients with greater $\mathrm{fMRI}$ signal increase in EBA tended to show greater reduction in the general psychopathology scale. The fMRI signal changes in EBA were neither correlated with changes in the positive $(r=0.11, P=0.73)$ nor negative scale of PANSS $(r=-0.48$, $P=0.095)$. The $\mathrm{fMRI}$ signal changes in EBA were not correlated with changes in BMI $(r=-0.01, P=0.964)$. Regression analysis revealed that none among gender, age, duration of illness and chlorpromazine-equivalent daily dosage was related to $\mathrm{fMRI}$ signal changes in EBA $(P>0.05)$. Confirmatory statistical parametric mapping (SPM) analysis revealed that the $\mathrm{FMRI}$ signal changes in EBA were negatively correlated with changes in the general psychopathology scale of PANSS (peak: $x=42, y=-74, z=4$, $Z$ score $=3.82$, cluster size: 7 voxels) (Figure 4 ).

\section{Discussion}

Exercise is being increasingly implemented for the management of obesity and weight gain in schizophrenia, $, 7,33$ and indeed the patients participating in the current program succeeded in reducing BMI. In addition, we demonstrated that sports participation also had a positive effect on psychiatric symptoms in schizophrenia.

A body of literature has shown that exercise has positive effects on cognitive functions in the general population throughout life, and even in patients with neurodegenerative diseases. ${ }^{11,34} \mathrm{~A}$ recent magnetic resonance imaging study demonstrated that improvement of short-term memory induced by exercise was associated with an increase in hippocampal volume in older adults. ${ }^{34}$ The beneficial effect of exercise on hippocampal volume and short-term memory was also demonstrated in chronic schizophrenia. ${ }^{12}$ 
In contrast, despite the fact that physical therapy and sports are widely implemented in treatment/rehabilitation of schizophrenia and are also known to have a positive effect on psychiatric symptoms of schizophrenia, ${ }^{9,10}$ little is known about the neurobiological mechanism of the effect. We found that basketball participation increased EBA activation during observation of basketball-related actions. Furthermore, the increase in EBA activation was associated with improvement in the general psychopathology scale of PANSS. Previous studies suggested that EBA integrates multisensory bodyrelated information (for example, internal action-related signal and external visual signal) ${ }^{35}$ and is involved in planning, execution, rehearsal and imagination of actions as part of a system for understanding the action of others (for example, MNS). ${ }^{22,36}$ It was reported that EBA activation was enhanced during imitation compared with observation of actions. ${ }^{22}$ The motivation to act or the preparation for action has also been shown to modulate EBA activity. ${ }^{37,38}$ Furthermore, several studies including ours have shown that EBA might contribute to the inferring the intention of others. ${ }^{23,39-41}$

Playing in team sports requires us to anticipate and understand the actions of team members and opponents in various contexts of the game. Increased EBA activation induced by basketball participation might reflect more appropriate integration of body-related information and contextual information of actions. Consequently, these restorations might mediate improvements in understanding others' intentions, interpersonal communication, body awareness, and overall physical activity manifested as the improvement in the general psychopathology scale. Intuitively, bodyrelated items of the general psychopathology scale such as somatic concern, tension, posturing, depression and motor retardation might be more directly linked to EBA functions. However, the general psychopathology scale is a complex construct, and the exact mechanism that links the increase of EBA activation and the improvement in the general psychopathology scale need to be elucidated in future investigations.

The current study has several limitations. First, this is not a randomized control study. The patients participating in the program might have had stronger motivations to improve their physical as well as mental conditions than the control group. Second, the patients' clinical backgrounds were heterogeneous. Although medications remained unchanged during this study, it is possible that antipsychotics might affect baseline brain activation even if the density of $\mathrm{D} 2$ receptors is extremely low in the posterior temporal-occipital cortex. ${ }^{42,43}$ Any generalization should therefore be approached with caution until we have some replications in larger samples. Third, we used basketball for intervention and visual stimuli. Although we do not think our finding about basketball-related action is generalizable to other sports, future studies will be needed to test whether an effect on brain activation is limited to the sport used in the program or is generalizable to other sports. Finally, follow-up studies are necessary to examine whether the observed positive effect is transient or longlasting. Notwithstanding these limitations, as a first step, we conducted the current study from a perspective of feasibility and real-life setting.
In conclusion, we demonstrated that sports participation had a positive effect not only on weight gain but also on psychiatric symptoms in schizophrenia. EBA, as a part of the system for understanding the intention and action of others, might mediate these beneficial effects of sports participation. The results of this study merit further investigation of the neurobiological mechanisms underlying the therapeutic effect of exercise/sports not only for schizophrenia but also other neuropsychiatric disorders.

\section{Conflict of interest}

The authors declare no conflict of interest.

Acknowledgements. This study was supported by a Health and Labor Sciences Research Grant for Research on Psychiatric and Neurological Diseases and Mental Health (H20-KOKORO-025) from the Japanese Ministry of Health, Labor and Welfare, a Grant-in-Aid for Scientific Research on Innovative Areas: Prediction and Decision Making (23120009), a Grant-in-Aid for Scientific Research (A) (23680045) MEXT, Japan, Takeda Science Foundation, Casio Science Foundation, and Senshin Medical Research Foundation. Mieko Mori and Harumi Murayama and the staff of the day-hospital unit of Asai Hospital are gratefully acknowledged for nursing care and assistance with subject recruitment.

1. Lan TH, Loh EW, Wu MS, Hu TM, Chou P, Lan TY et al. Performance of a neuro-fuzzy model in predicting weight changes of chronic schizophrenic patients exposed to antipsychotics. Mol Psychiatry 2008; 13: 1129-1137.

2. Marder SR, Essock SM, Miller AL, Buchanan RW, Casey DE, Davis JM et al. Physical health monitoring of patients with schizophrenia. Am J Psychiatry 2004; 161: 1334-1349.

3. Correll CU, Manu P, Olshanskiy V, Napolitano B, Kane JM, Malhotra AK. Cardiometabolic risk of second-generation antipsychotic medications during first-time use in children and adolescents. JAMA 2009; 302: 1765-1773.

4. Girgis RR, Javitch JA, Lieberman JA. Antipsychotic drug mechanisms: links between therapeutic effects, metabolic side effects and the insulin signaling pathway. Mol Psychiatry 2008; 13: 918-929.

5. Nasrallah HA. Atypical antipsychotic-induced metabolic side effects: insights from receptorbinding profiles. Mol Psychiatry 2008; 13: 27-35.

6. Faulkner G, Soundy AA, Lloyd K. Schizophrenia and weight management: a systematic review of interventions to control weight. Acta Psychiatr Scand 2003; 108: 324-332.

7. Vancampfort D, Knapen J, De Hert M, van Winkel R, Deckx S, Maurissen K et al. Cardiometabolic effects of physical activity interventions for people with schizophrenia. Phys Ther Rev 2009; 14: 388-398.

8. Friedman Jl, Wallenstein S, Moshier E, Parrella M, White L, Bowler S et al. The effects of hypertension and body mass index on cognition in schizophrenia. Am J Psychiatry 2010; 167: $1232-1239$

9. Langle G, Siemssen G, Hornberger S. Role of sports in treatment and rehabilitation of schizophrenic patients. Rehabilitation (Stuttg) 2000; 39: 276-282.

10. Vancampfort D, Probst M, Helvik Skjaerven L, Catalan-Matamoros D, Lundvik-Gyllensten A, Gomez-Conesa A et al. Systematic review of the benefits of physical therapy within a multidisciplinary care approach for people with schizophrenia. Phys Ther 2012; 92: 11-23.

11. Hillman $\mathrm{CH}$, Erickson $\mathrm{KI}$, Kramer AF. Be smart, exercise your heart: exercise effects on brain and cognition. Nat Rev Neurosci 2008; 9: 58-65.

12. Pajonk FG, Wobrock T, Gruber O, Scherk H, Berner D, Kaizl I et al. Hippocampal plasticity in response to exercise in schizophrenia. Arch Gen Psychiatry 2010; 67: 133-143.

13. Astafiev SV, Stanley CM, Shulman GL, Corbetta M. Extrastriate body area in human occipital cortex responds to the performance of motor actions. Nat Neurosci 2004; 7 : 542-548.

14. Grezes J, Decety J. Functional anatomy of execution, mental simulation, observation, and verb generation of actions: a meta-analysis. Hum Brain Mapp 2001; 12: 1-19.

15. Grossman ED, Blake R. Brain activity evoked by inverted and imagined biological motion. Vision Res 2001; 41: 1475-1482.

16. lacoboni M, Dapretto M. The mirror neuron system and the consequences of its dysfunction. Nat Rev Neurosci 2006; 7: 942-951.

17. Rizzolatti G, Craighero L. The mirror-neuron system. Annu Rev Neurosci 2004; 27: 169-192.

18. Bonini L, Ferrari PF. Evolution of mirror systems: a simple mechanism for complex cognitive functions. Ann N Y Acad Sci 2011; 1225: 166-175.

19. Buccino G, Amore M. Mirror neurons and the understanding of behavioural symptoms in psychiatric disorders. Curr Opin Psychiatry 2008; 21: 281-285. 
20. Takahashi H, Kato M, Sassa T, Shibuya T, Koeda M, Yahata N et al. Functional deficits in the extrastriate body area during observation of sports-related actions in schizophrenia. Schizophr Bull 2010; 36: 642-647.

21. Downing PE, Jiang $Y$, Shuman M, Kanwisher N. A cortical area selective for visual processing of the human body. Science 2001; 293: 2470-2473.

22. Jackson PL, Meltzoff AN, Decety J. Neural circuits involved in imitation and perspectivetaking. Neuroimage 2006; 31: 429-439.

23. Takahashi H, Shibuya T, Kato M, Sassa T, Koeda M, Yahata N et al. Enhanced activation in the extrastriate body area by goal-directed actions. Psychiatry Clin Neurosci 2008; 62: 214-219.

24. Calvo-Merino B, Glaser DE, Grezes J, Passingham RE, Haggard P. Action observation and acquired motor skills: an FMRI study with expert dancers. Cereb Cortex 2005; 15: 1243-1249.

25. Cross ES, Hamilton AF, Grafton ST. Building a motor simulation de novo: observation of dance by dancers. Neuroimage 2006; 31: 1257-1267.

26. Borg GA. Psychophysical bases of perceived exertion. Med Sci Sports Exerc 1982; 14 377-381.

27. Haskell WL, Lee I, Pate RR, Powell KE, Blair SN, Franklin BA et al. Physical activity and public health: updated recommendation for adults from the American College of Sports Medicine and the American Heart Association. Med Sci Sports Exerc 2007; 39: 1423-1434.

28. American Psychiatric Association. Diagnostic and Statistical Manual of Mental Disorders, 4th edn. American Psychiatric Association: Washington, DC, 1994

29. Rey MJ, Schulz P, Costa C, Dick P, Tissot R. Guidelines for the dosage of neuroleptics. I: Chlorpromazine equivalents of orally administered neuroleptics. Int Clin Psychopharmacol 1989; 4: 95-104.

30. Rijcken CA, Monster TB, Brouwers JR, de Jong-van den Berg LT. Chlorpromazine equivalents versus defined daily doses: how to compare antipsychotic drug doses? J Clin Psychopharmacol 2003; 23: 657-659.

31. First M, Spitzer R, Gibbon M. Structured Clinical Interview forDSM-IV Axis I Disorders Patient Edition (SCID-I/P, Version 2.0). Biometrics Research Department, New York State Psychiatric Institute: New York, 1995.

32. Kay SR, Fiszbein A, Opler LA. The positive and negative syndrome scale (PANSS) for schizophrenia. Schizophr Bull 1987; 13: 261-276.
33. Faulkner G, Cohn T, Remington G. Interventions to reduce weight gain in schizophrenia Schizophr Bull 2007; 33: 654-656.

34. Erickson KI, Voss MW, Prakash RS, Basak C, Szabo A, Chaddock L et al. Exercise training increases size of hippocampus and improves memory. Proc Natl Acad Sci USA 2011; 108 : 3017-3022.

35. Arzy S, Thut G, Mohr C, Michel CM, Blanke O. Neural basis of embodiment: distinct contributions of temporoparietal junction and extrastriate body area. J Neurosci 2006; 26: 8074-8081.

36. Jeannerod M. Visual and action cues contribute to the self-other distinction. Nat Neurosci 2004; 7: 422-423.

37. Cheng Y, Meltzoff AN, Decety J. Motivation modulates the activity of the human mirrorneuron system. Cereb Cortex 2007; 17: 1979-1986.

38. Kuhn S, Keizer A, Rombouts SA, Hommel B. The functional and neural mechanism of action preparation: roles of EBA and FFA in voluntary action control. $J$ Cogn Neurosci 2011; 23: 214-220.

39. Amoruso L, Couto B, Ibanez A. Beyond extrastriate body area (EBA) and fusiform body area (FBA): context integration in the meaning of actions. Front Hum Neurosci 2011; 5: 124

40. Marsh AA, Kozak MN, Wegner DM, Reid ME, Yu HH, Blair RJ. The neural substrates of action identification. Soc Cogn Affect Neurosci 2010; 5: 392-403.

41. Zimmermann M, Meulenbroek RG, de Lange FP. Motor planning is facilitated by adopting an action's goal posture: an fMRI study. Cereb Cortex 2012; 22: 122-131.

42. Takahashi H, Higuchi M, Suhara T. The role of extrastriatal dopamine D2 receptors in schizophrenia. Biol Psychiatry 2006; 59: 919-928.

43. Takahashi $\mathrm{H}$, Matsui $\mathrm{H}$, Camerer $\mathrm{C}$, Takano H, Kodaka F, Ideno $\mathrm{T}$ et al. Dopamine D receptors and nonlinear probability weighting in risky choice. J Neurosci 2010; 30 16567-16572.

Translational Psychiatry is an open-access journal published by Nature Publishing Group. This work is licensed under the Creative Commons Attribution-Noncommercial-No Derivative Works 3.0 Unported License. To view a copy of this license, visit http://creativecommons.org/licenses/by-nc-nd/3.0/ 